\title{
Prevalencia de malnutricion cronica y anemia en niños de 6 a 36 meses en el distrito de sarhua, Ayacucho
}

\author{
Prevalence of chronic malnutrition and anemia in children from 6 to 36 months in the district of \\ Sarhua, Ayacucho
}

\author{
Enrique Morales M. ${ }^{1}$, María E. Villanueva E. ${ }^{2}$; Kruskaya Quispe A. ${ }^{3}$
}

\begin{abstract}
Resumen
El objetivo del presente estudio fue evaluar el estado de nutrición de niños de 6 a 36 meses del distrito de Sarhua, departamento de Ayacucho. Se usaron los siguientes métodos: 1) Antropométrico: indicadores talla/longitud para edad (malnutrición crónica); peso para talla/longitud (malnutrición aguda) y peso para edad (malnutrición global). 2) o Bioquímico: método de la cianometahemaglobina para determinación de hemoglobina y 3) Encuesta de recordatorio de 24 horas. El estudio fue transversal con una muestra de 43 niños seleccionados por muestreo bietápico-probabilístico, realizando una encuesta basal de calificación previa. Los resultados mostraron un 44,2\% de niños normales y 55,8 \% con malnutrición crónica; de estos, 34,9 \% tuvieron MC moderada: entre -2 a -3 Desviaciones Estándar (DE) y 20,9 \% tuvieron MC severa, por debajo de $<.3 \mathrm{DE}$. No hubo niños con desnutrición aguda, pero hubo $16,27 \%$ de niños con riesgo de sobrepeso $(\mathrm{P} / \mathrm{T}$ entre $+1 \mathrm{y}+2 \mathrm{DE})$. La prevalencia de anemia fue de $34,87 \%$. La dieta de los niños a partir de los seis meses de edad estuvo constituida por lactancia materna y alimentación complementaria (AC), con una ingesta calórica significativamente mayor a lo recomendado por FAO; el mayor porcentaje de calorías totales fue proporcionado por carbohidratos, mientras que las grasas contribuyeron solo con $14 \%$ de la energía total y las proteínas $8 \%$ en promedio. También hubo marcada deficiencia de ingesta de micronutrientes como hierro y zinc. El consumo de vitamina A excedió al requerimiento. Los resultados del presente estudio revelan una alta prevalencia de malnutrición crónica, $55,8 \%$, y de anemia, $34,8 \%$, causadas principalmente por déficit de ingesta de grasas y de hierro y zinc. Esta situación podría atribuirse, entre otras causas, al consumo de una dieta familiar basada principalmente en productos de origen vegetal ricos en carbohidratos como son los cereales y tubérculos, con escasa presencia de alimentos de origen animal. Palabras clave: malnutrición crónica; anemia; distrito Sarhua; consumo de alimentos.
\end{abstract}

\begin{abstract}
The objective of this study was to evaluate the nutritional status of children from 6 to 36 months of age in the District of Sarhua, Department of Ayacucho. The following methods were used: 1) anthropometry: high /length indicators for age (chronic malnutrition); weight for height/length (acute malnutrition) and weight for age (global malnutrition). 2) Biochemical: Hemoglobin was measured by the cianometahemoglobin method and 3) 24 hours reminder survey for food intake. A cross-sectional study was conducted with a sample of 43 children selected by two-stage probabilistic sampling. The results showed that 44,2 \% had a normal nutrition and 55,8\% had chronic malnutrition (CM), from these, 34,9\% had moderate CM (-2 to $-3 \mathrm{SD})$, and 20,9\% had severe CM (<-3 SD). There were no children with acute malnutrition but we found $16,27 \%$ of children with risk of overweight $(\mathrm{W} / \mathrm{H}+1$ to $+2 \mathrm{SD})$. The prevalence of anemia was $34,87 \%$. The diet of these children from 6 to 36 months was based on breast feeding and complementary food (CF) with a caloric intake significantly higher than the daily intake recommended by FAO. Carbohydrates contributed with the highest percentage of the total calories, while fat contributed only $14 \%$ of the total energy, and proteins represented only $8 \%$ in average. There were also marked deficiencies of micronutrients such as iron and zinc. The intake of vitamin A exceeded the daily requirements. The results from this study reveal a high prevalence of chronic malnutrition, 55,8 \% and anemia, 34,8 \%, caused mainly by an intake deficit of fat, iron and zinc. This situation could be attributed, among other things, to the consumption of a diet based mainly on vegetables rich in carbohydrates such as cereals and tubers, with low presence of food of animal origin.
\end{abstract}

Keywords: chronic malnutrition; anemia; Sarhua district; food consumption.

\footnotetext{
Docente Asociado, Departamento de Nutrición. Universidad Nacional Agraria La Molina, Lima, Perú. Email: emorales@lamolina.edu.pe Docente Principal, Departamento de Nutrición. Universidad Nacional Agraria La Molina, Lima, Perú. Email: mvillanueva@lamolina.edu.pe Maestrando, Especialidad de Nutrición. Universidad Nacional Agraria La Molina, Lima, Perú.
} 


\section{Introducción}

La nutrición y salud de los niños menores de tres años es de suma importancia debido a que es la etapa de la vida de mayor velocidad de crecimiento, en consecuencia, sus requerimientos nutricionales son mayores que en edades posteriores. En los países en vías de desarrollo, dentro de los que se encuentra el Perú, las deficiencias de consumo de alimentos, así como la presencia de enfermedades son de alta prevalencia en estos niños y traen consecuencias negativas, afectando el crecimiento, desarrollo, rendimiento intelectual y la capacidad laboral en la vida adulta (Brown \& Pollit, 1996).

El estado de nutrición de los niños está vinculado al desarrollo cognitivo, al proceso de aprendizaje y al rendimiento escolar (Leiva et al., 2001). Asimismo, está asociado a la capacidad de respuesta frente a las enfermedades porque un inadecuado estado nutricional incrementa tanto la morbilidad como la mortalidad en la infancia. Los efectos de la nutrición se prolongan a lo largo de la vida, ya que incrementan el riesgo de padecer enfermedades crónicas (sobrepeso, obesidad, diabetes, enfermedades cardiovasculares) (Jason, 2004) y se relacionan con menores logros educativos y económicos en la adultez.

Actualmente, el estado nutricional de los niños es empleado en el ámbito internacional como parte de los indicadores del desarrollo de los países. Por ello, forma parte de los Objetivos de Desarrollo del Milenio junto con otros indicadores de desarrollo social y económico.

La anemia por deficiencia de hierro en los menores de cinco años es otro de los problemas de nutrición de mayor magnitud en el Perú, en especial en los departamentos de la sierra, como Ayacucho y sus respectivas provincias, donde las prevalencias alcanzan valores entre 33 y $72 \%$ para niños entre 6 y 35 meses y los más pequeños son los más afectados (ENDES, 2010).

Sarhua no es ajena a esta problemática ya que se encuentra registrada como uno de los distritos en extrema pobreza y exclusión social de acuerdo con el Instituto Nacional de Estadística e Informática; encontramos elevada prevalencia de malnutrición crónica y anemia, las cuales contribuyen a deteriorar el estado de la salud de la comunidad y constituyen un factor limitante para el desarrollo de sus capacidades como grupo humano (INS, 2003; WHO, 2001).

La deficiencia de micronutrientes como vitamina A y hierro se presenta a nivel nacional, especialmente en las zonas en las que es escaso el consumo de frutas y verduras, donde los niños de seis meses a dos años presentan anemia, retraso en el crecimiento físico, bajo desarrollo de la capacidad cognitiva y menor resistencia a las enfermedades infecciosas (Pollit, 1997).

El objetivo del presente estudio fue determinar, en el distrito de Sarhua, provincia de Víctor Fajardo, Ayacucho, la prevalencia de desnutrición crónica y anemia, así como también evaluar las características de ingesta de energía, macronutrientes y micronutrientes a través de encuestas de consumo de alimentos por recordatorio de 24 horas y la relación entre estos factores, en niños de 6 a 36 meses, grupo señalado como prioritario en la Estrategia Nacional de Seguridad Alimentaria del Perú 2004-2015 (PCM, 2004).

\section{Materiales y métodos}

\section{Tipo y área de estudio}

Este fue un estudio descriptivo transversal realizado en el departamento de Ayacucho, provincia de Víctor Fajardo, distrito de Sarhua, cuya recolección de información se realizó entre los meses de febrero y marzo del 2008.

Sarhua es una comunidad campesina rural dedicada a la agricultura y pastoreo de autoabastecimiento. Se encuentra ubicada al Noreste de la provincia de Víctor Fajardo, a una altitud de $3.389 \mathrm{msnm}$, con una población de 3.043 habitantes y cuenta con seis centros poblados, cuatro establecimientos de salud y ocho instituciones educativas.

\section{Población y muestra}

La selección de la muestra se realizó por conglomerados, en forma sistemática, en tres etapas y aleatoria; estuvo conformada por 43 hogares, lo que significó 43 niños y niñas de 6 a 36 meses, con edades entre 6 y 12 meses, 6 individuos (14\%), entre 12,1 y 24 meses, 23 individuos $(53,5 \%)$ y entre 24,1 y 36 meses, 14 individuos $(32,5$ $\%)$. Las familias, estuvieron conformadas por dos a ocho miembros, media $5(=4,67)$ por familia. El tamaño de la muestra se calculó tomando como referencia la prevalencia de malnutrición crónica en niños menores de cinco años (43,5 \% correspondiente al departamento de Ayacucho, con un nivel de confianza de $95 \%$ ). La muestra correspondió al $60 \%$ de los hogares con la característica de inclusión, según datos de una encuesta basal. Se excluyeron a quienes presentaron problemas o defectos físicos que impedían realizar una adecuada medición de peso o talla. Esta población es bilingüe (quechua/castellano) por lo cual se contrató a una enfermera de la zona que sirvió de intérprete.

\section{Estado de nutrición}

El estado de nutrición de los niños menores de tres años se informa a través de los indicadores de uso internacional: malnutrición crónica (talla/edad), malnutrición aguda (peso/talla) y malnutrición global (peso/edad) según sexo de niñas y niños. La clasificación del estado de nutrición se realizó en base al Patrón de Crecimiento Infantil de la Organización Mundial de la Salud (OMS) difundido internacionalmente el año 2006, según puntaje Z, Malnutrición Crónica (MC) total $(<2 \mathrm{DE})$; MC moderada (entre -2 y -3 DE) y MC severa (<-3 DE) (Who, 2001; OMS, 1995; OPS, 1991). La medición de la hemoglobina se realizó con una lectora portátil Hemocue $₫$. (usa método de cianometahemoglobina). Se ajustaron las mediciones de hemoglobina en función de la altitud empleando el método de la Encuesta Nacional de Demografía y Salud (ENDES 2010), para determinar el estado de nutrición de hierro (hemoglobina).

\section{Consumo de alimentos}

Para determinar las características del consumo de alimentos, se realizó una encuesta de recordatorio de 24 horas dirigida a la madre de familia o bien a la persona 
que provee los alimentos en el hogar; se solicitó que respondieran qué alimentos consumieron los niños un día anterior a la encuesta, se anotó las preparaciones especificando el tipo de cocción, para luego registrar los principales ingredientes y luego las cantidades, con ayuda de un laminario de medidas caseras (CENAN, 2007; PRISMA, 1996).

\section{Aspectos éticos}

Antes del inicio de la recolección de la información, se solicitó dos tipos de consentimiento: en primer lugar, el comunitario, representado por el Teniente Alcalde quien era la máxima autoridad durante el desarrollo del trabajo y a quien se brindó los detalles de la actividad y, en segundo lugar, se procedió a obtener el consentimiento informado de las madres, a quienes se les solicitó la firma de un documento aprobado por el Comité de Ética del Instituto de Investigación Nutricional. El texto del consentimiento fue leído por el encuestador en castellano o en quechua, según fuera necesario.

De acuerdo con los resultados, se derivó al centro de salud de la zona a aquellas personas que se encontraban con anemia para el respectivo tratamiento; además, se brindó una orientación nutricional sobre alimentación y nutrición de acuerdo con cada diagnóstico.

\section{Análisis de datos.}

Se procedió a digitar la información desde los registros físicos a una base de datos, utilizando el paquete estadístico
SPSS v13. El análisis estadístico comprende el cálculo de la prevalencia del estado nutricional de la población objetivo, sus intervalos de confianza al $95 \%$ y la frecuencia de consumo de los principales alimentos. Se cuantificó la asociación entre malnutrición crónica, anemia y factores como edad, usando para ello la prueba del Chi cuadrado, para ello se incluyó variables que fueron seleccionadas considerando su relevancia para los objetivos de la investigación.

\section{Resultados y discusión \\ Estado de nutrición de los niños \\ Antropometría}

El estado de nutrición de los niños, determinado según el indicador talla/longitud para edad $(\mathrm{T} / \mathrm{E})$, expresado en puntaje $\mathrm{Z}$ (Tabla 1), muestra que del total de niños estudiados, $43(100 \%)$ se clasificaron como nutrición normal: de $-2 \mathrm{a}+2 \mathrm{DE}=19$ (44,2 \%), como malnutridos crónicos $(\mathrm{MC})=24(55,8 \%)$; de estos, 15 (34,9\%) tuvieron MC moderada: entre -2 y -3 DE y 9 (20,9\%) tuvieron MC severa: por debajo de $-3 \mathrm{DE}$.

La distribución de los puntajes $\mathrm{Z}$ de la talla/edad de niños presentó un mínimo de -- 4,60 DE y un máximo de $+0,98$ DE, con una media de $-2,1063$ y una D.E. de 1,17359 .

La Fig. 1 muestra la distribución de los puntajes $\mathrm{Z}$ para la relación talla/ edad y en ella se puede observar que la media se ubica entre -2 y -3 D.E.; los niños evaluados en

Tabla 1. Estado de nutrición, según indicador talla/longitud para edad (T/E) de los niños de 6 a 36 meses de edad

\begin{tabular}{lcll}
\hline Estado de Nutrición & Puntaje Z & Número & $\%$ \\
Normal & de $-2 \mathrm{a}+2 \mathrm{DE}$ & 19 & 44,2 \\
Malnutrición Crónica (MC) & $<-2$ DE & 24 & 55,8 \\
\cline { 1 - 4 } MC moderada & de -2 a -3 DE & $(15)$ & $(34,9)$ \\
\cline { 1 - 1 } MC severa & $<-3$ DE & $(9)$ & $(20,9)$ \\
\hline Total & & 43 & 100 \\
\hline
\end{tabular}

Desviación estándar (DE)

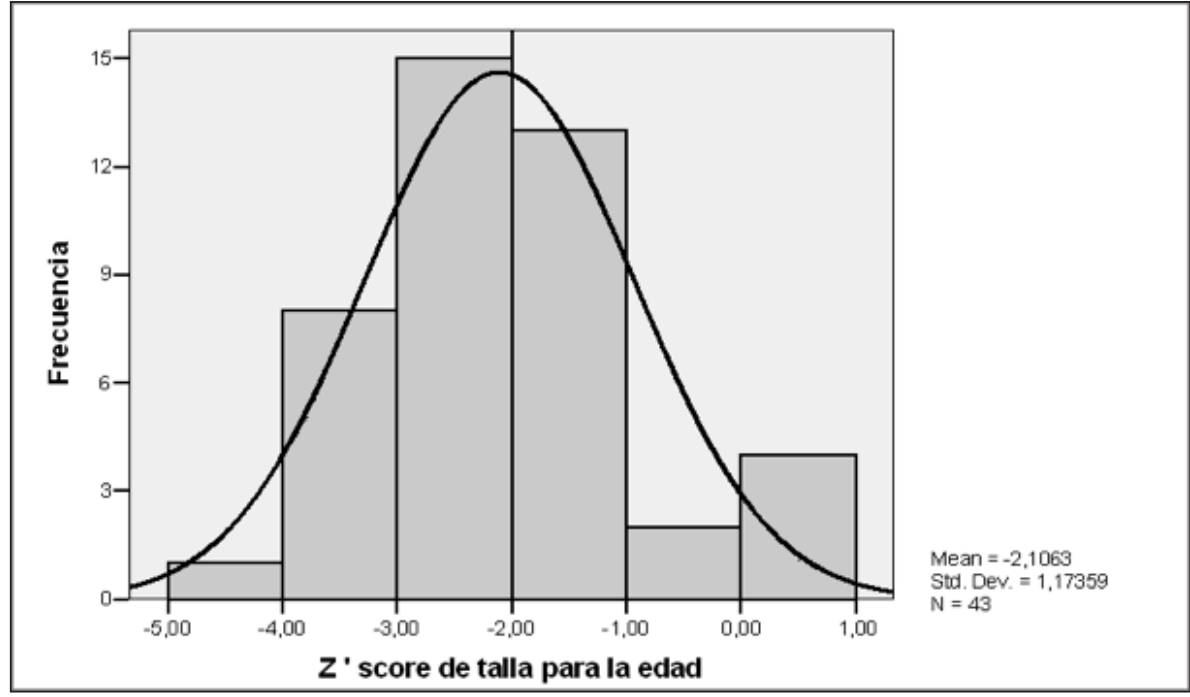

Figura 1. Distribución de puntaje $Z$ de $T / E$ en niños de 6 a 36 meses de edad del distrito de Sarhua, Ayacucho 2008 
su mayoría, efectivamente, sufren de malnutrición crónica. La malnutrición crónica afecta a niños y niñas, presentándose con mayor prevalencia en niños entre 6 y 12 meses. No hay malnutrición aguda (peso/talla), más bien hay $16,28 \%$ de niños con riesgo de sobrepeso, entre +1 $\mathrm{y}+2 \mathrm{DE}$ de $\mathrm{P} / \mathrm{T}$. El nivel educativo de la madre influye en la malnutrición crónica presentándose $60 \%$ en madres sin estudios, esto sugiere que a pesar de un período largo de adaptación a insuficiente oferta alimentaria hay hábitos alimentarios inadecuados que llevan a un sobrepeso.

\section{Anemia}

La anemia es un problema nutricional de mucha preocupación, especialmente en las zonas rurales de nuestro país y en niños menores de tres años. La medición de la hemoglobina en los niños presentó valores entre 7 y $15,6 \mathrm{mg} / \mathrm{dL}$, una media de $11,409 \mathrm{mg} / \mathrm{dL}$ y desviación estándar de 1,7945 como se muestra en la Fig. 2.

La prevalencia de anemia (Tabla 2) fue de 34,87\% (15 de 43 niños evaluados tuvieron algún grado de anemia), el $20,93 \%$ presentó anemia leve, el 11,62 \% anemia moderada

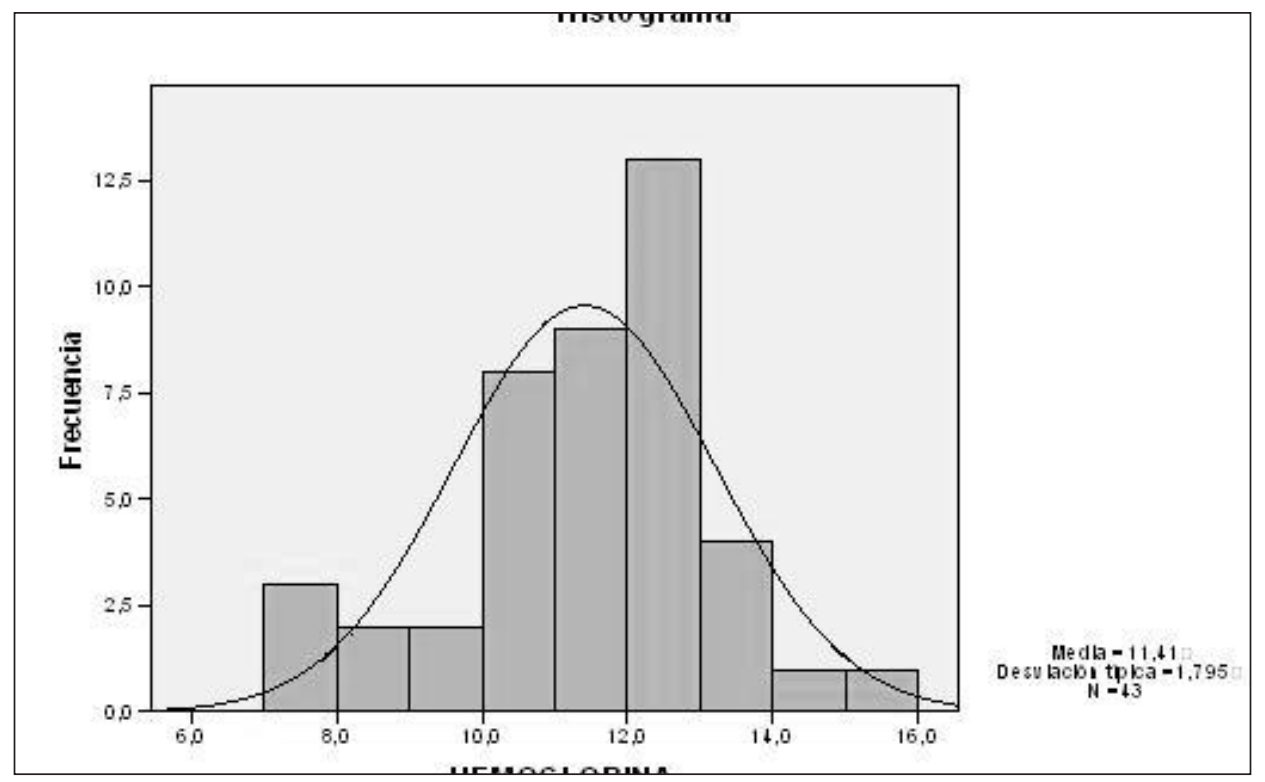

Figura 2. Histograma de frecuencias de hemoglobina del niño evaluado (mg/dl)

Tabla 2: prevalencia de anemia en niños entre 6 a 36 meses

\begin{tabular}{|c|c|c|c|c|c|c|c|c|c|c|}
\hline \multirow{3}{*}{$\begin{array}{l}\text { CARACTERÍSTICA } \\
\text { Edad del niño }\end{array}$} & \multicolumn{6}{|c|}{$\begin{array}{l}\text { DISTRIBUCIÓN PORCENTUAL DE NIÑOS CON } \\
\text { PRUEBA DE ANEMIA }\end{array}$} & \multirow{2}{*}{\multicolumn{2}{|c|}{$\begin{array}{l}\text { NIÑOS SIN } \\
\text { ANEMIA } \\
\text { Total }\end{array}$}} & \multirow{2}{*}{\multicolumn{2}{|c|}{$\begin{array}{l}\text { NUMERO DE } \\
\text { NIÑOS } \\
\%\end{array}$}} \\
\hline & \multicolumn{2}{|c|}{ ANEMIA SEVERA } & \multicolumn{2}{|c|}{$\begin{array}{l}\text { ANEMIA } \\
\text { MODERADA }\end{array}$} & \multicolumn{2}{|c|}{$\begin{array}{l}\text { ANEMIA } \\
\text { LEVE }\end{array}$} & & & & \\
\hline & $\mathrm{N}^{\mathrm{o}}$ & $\%$ & $\mathrm{~N}^{\mathrm{o}}$ & $\%$ & $\mathrm{~N}^{\mathrm{o}}$ & $\%$ & $\mathrm{~N}^{\circ}$ & $\%$ & & \\
\hline $6-12$ meses & 1 & 16,67 & 2 & 33,33 & 1 & 16,67 & 2 & 33,33 & 6 & 100 \\
\hline $12,1-24$ meses & 0 & 0,00 & 3 & 13,04 & 5 & 21,74 & 15 & 65,22 & 23 & 100 \\
\hline $24,1-36$ meses & 0 & 0,00 & 0 & 0,00 & 3 & 21,43 & 11 & 78,57 & 14 & 100 \\
\hline TOTAL & 1 & 2,32 & 5 & 11,62 & 9 & 20,93 & 28 & 65,11 & 43 & 100 \\
\hline \multicolumn{11}{|l|}{ Sexo del niño } \\
\hline Hombre & 0 & 0 & 4 & 18,18 & 4 & 18,18 & 14 & 63,64 & 22 & 100 \\
\hline Mujer & 1 & 4,76 & 1 & 4,76 & 5 & 23,81 & 14 & 66,67 & 21 & 100 \\
\hline TOTAL & 1 & 2,32 & 5 & 11,62 & 9 & 20,93 & 28 & 65,11 & 43 & 100 \\
\hline \multicolumn{11}{|l|}{$\begin{array}{l}\text { Nivel educativo de la } \\
\text { madre }\end{array}$} \\
\hline Sin estudios & 0 & 0 & 0 & 0 & 1 & 20 & 4 & 80 & 5 & 100 \\
\hline Primaria & 1 & 3,84 & 2 & 7,69 & 5 & 19,23 & 18 & 69,23 & 26 & 100 \\
\hline Secundaria & 0 & 0 & 3 & 25 & 3 & 25 & 6 & 50 & 12 & 100 \\
\hline TOTAL & 1 & 2,32 & 5 & 11,62 & 9 & 20,93 & 28 & 65,11 & 43 & 100 \\
\hline
\end{tabular}


y el $2,32 \%$ anemia severa, esto último se presentó en un niño de $6-12$ meses.

En la Tabla 2 se puede apreciar que la prevalencia de anemia leve fue de $16,67 \%$, en un niño del grupo de 6 a 12 meses, de $21,74 \%$ en niños de 12 a 24 meses y $21,43 \%$ en niños de 24 a 36 meses. Se halló una asociación estadísticamente significativa entre la edad y la gravedad de la anemia; en niños de 6 a 12 meses predominó la anemia severa y moderada, mientras que en niños de 12,1 a 24 meses, la anemia moderada y leve; en los niños de 24,1 a 36 meses, solo se presentó anemia leve.

\section{Consumo de alimentos por recordatorio de 24 horas}

Los resultados de la evaluación de ingesta dietética, por recordatorio de 24 horas, indicaron que la alimentación complementaria diaria de los niños en estudio sobrepasa los requerimientos de energía recomendados, pero no cubre los requerimientos en micro nutrientes (FAO/OMS, 2004).

Al analizar la ingesta dietética por recordatorio de 24 horas y frecuencia de consumo de alimentos, se observó un promedio de consumo de energía de la alimentación complementaria: $382 \mathrm{kcal}$ para los niños entre 6 y 8 meses, $507,1 \mathrm{kcal}$ para niños entre 9 y 11 meses, $707 \mathrm{kcal}$ para niños de 12 a 36 meses (Fig. 3), superior a lo requerido en la ingesta de energía con relación a los valores recomendados por la FAO/OMS/UNU (2004).

El porcentaje de adecuación de la energía total a los requerimientos diarios de alimentación complementaria fue de $133 \%$ para niños de 6 a 8 meses, esto significa un $33 \%$ de energía por encima de los valores recomendados por la FAO/OMS/UNU (2004); 146,9 \% para niños de 9 a11 meses y 136,1 \% para niños de 12 a 23 meses; estos valores se encuentran por encima del promedio de consumo de energía recomendados por la FAO/OMS/ UNU (2004). Al analizar la composición de la dieta se observó que la mayor proporción de energía fue aportada por carbohidratos (cereales como maíz, trigo, cebada y tubérculos, como papa).

Analizando el contenido de los macro nutrientes, como porcentaje de la energía total, se observó que los carbohidratos aportaron en promedio: para niños de $6-8$ meses, 73,43 g $(293,72 \mathrm{kcal}$ y 76,89 \% de la energía total de la dieta), para niños de 9 a 11 meses 99,2 g (396,8 kcal y $78,24 \%$ de la energía total de la dieta) y para niños de 12 a 36 meses, 130,12 g (520,48 kcal y 73,57 \% de la energía total de la dieta).

Con relación a las grasas, se observó una baja ingesta dietaria durante el periodo de estudio; el aporte promedio para niños de $6-8$ meses fue de 6 g (54 kcal y 14,13\% de la energía total de la dieta), para niños de 9-11 meses de 7,40 g $(69,48 \mathrm{kcal}$ y $13,7 \%$ de la energía total de la dieta) y para niños de 12 a 36 meses 13,14 g (118,26 kcal y 16,71\% de la energía total de la dieta). Los porcentajes de adecuación variaron de acuerdo con la la edad, observándose ingesta de grasa inferior a la recomendada, cuando tanto la cantidad como la calidad de las grasas ingeridas pueden afectar el desarrollo y crecimiento de los niños.

Durante el periodo de alimentación complementaria y por lo menos hasta los 24 meses, la alimentación de los niños debería tener entre 30 y $40 \%$ de la energía como grasa y aportar niveles de ácidos grasos esenciales, similares a los que se encuentran en la leche materna (Deweyet al., 1998). Con respecto a las proteínas totales consumidas, el aporte excede a los requerimientos en $6,87 \%$ para niños de 9 a 11 meses y de $19 \%$ para niños de 12 a 36 meses, pero hay un déficit de 19,78\% para niños de 6 a 8 meses. Para el cálculo de adecuación de proteínas se consideró los requerimientos de $9,1 \mathrm{~g}$ /día para niños de 6 a 8 meses, 9,6 $\mathrm{g} /$ día para niños de 9 a 11 meses y $10,9 \mathrm{~g}$ /día para niños de 12 a 36 meses, según los requerimientos de FAO/OMS (2004). Analizando la procedencia de la fuente proteica de la dieta, se observó que el 16,65\% fue de origen animal.

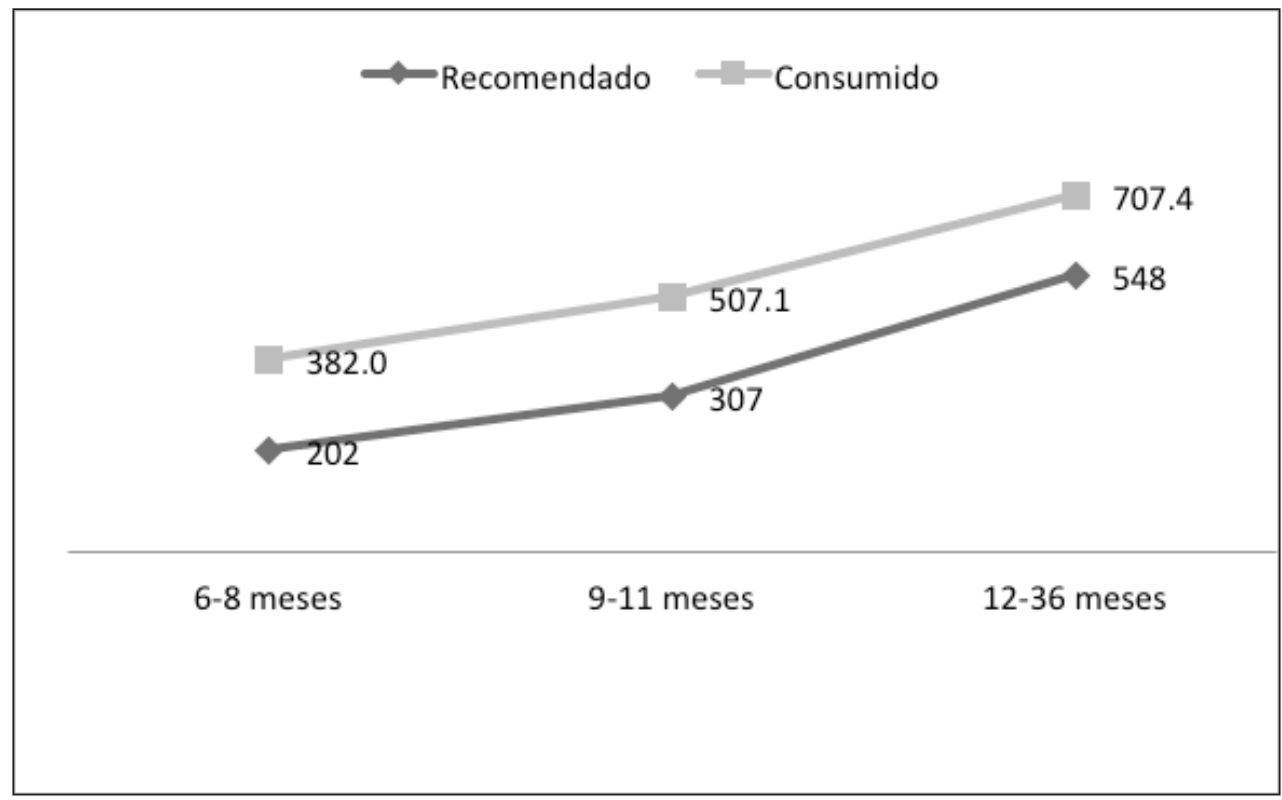

Figura 3. Comparación entre energía requerida y consumida de alimentos complementarios (kcal) 
Tabla 3. Requerimientos y consumo de alimentos en niños de 6 a 8 meses

\begin{tabular}{|c|c|c|c|c|c|c|c|c|}
\hline & $\begin{array}{c}\text { Calcio } \\
\text { mg }\end{array}$ & $\begin{array}{c}\text { Zinc } \\
\text { mg }\end{array}$ & $\begin{array}{c}\text { Hierro } \\
\mathrm{mg}\end{array}$ & $\begin{array}{c}\text { Retinol } \\
\mu \mathrm{g}\end{array}$ & $\begin{array}{c}\text { Tiamina } \\
\mathrm{mg}\end{array}$ & $\begin{array}{l}\text { Riboflavina } \\
\text { mg }\end{array}$ & $\begin{array}{l}\text { Niacina } \\
\mathrm{mg}\end{array}$ & $\begin{array}{c}\text { Vit C } \\
\mathrm{mg}\end{array}$ \\
\hline Requerimiento & 400 & 2,8 & 9,3 & 375 & 0,2 & 0,4 & 2 & 25 \\
\hline Alimento complementario & 198,9 & 1,36 & 4,4 & 88,9 & 0,3 & 0,2 & 3,5 & 17,3 \\
\hline Lactancia materna & 461,6 & 1,03 & 0,4 & 370,5 & 0,2 & 0,4 & 0,6 & 15,2 \\
\hline Total & 660,4 & 2,4 & 4,8 & 459,4 & 0,5 & 0,6 & 4,1 & 32,4 \\
\hline \% Adecuación & 165,1 & 85,4 & 51,3 & 122,5 & 243,9 & 147,6 & 205,5 & 129,8 \\
\hline
\end{tabular}

Tabla 4. Requerimientos y consumo de alimentos en niños de 9 a 11 meses

\begin{tabular}{|l|l|l|l|l|l|l|l|l|}
\hline \multicolumn{1}{c|}{} & Calcio & Zinc & Hierro & Retinol & Tiamina & Riboflavina & Niacina & Vit C \\
\cline { 2 - 10 } \multicolumn{1}{c|}{} & $\mathrm{mg}$ & $\mathrm{mg}$ & $\mathrm{mg}$ & $\mathrm{ug}$ & $\mathrm{mg}$ & $\mathrm{mg}$ & $\mathrm{mg}$ & $\mathrm{mg}$ \\
\hline Requerimiento & 400 & 4,1 & 9,3 & 400 & 0,3 & 0,4 & 4 & 30 \\
\hline $\begin{array}{l}\text { Alimento } \\
\text { complementario }\end{array}$ & 255 & 2 & 6,6 & 240 & 0,2 & 0,5 & 6,7 & 41,9 \\
\hline Lactancia materna & 424 & 0,95 & 0,4 & 340 & 0,17 & 0,33 & 0,56 & 13,93 \\
\hline Total & 679 & 2,95 & 7,03 & 580 & 0,42 & 0,82 & 7,3 & 55,84 \\
\hline \% Adecuación & 170 & 71,9 & 75,6 & 145 & 138,6 & 205,1 & 182,4 & 186,1 \\
\hline
\end{tabular}

Tabla 5. Requerimientos y consumo de alimentos en niños de 12 a 36 meses

\begin{tabular}{|l|r|r|r|r|r|r|r|r|}
\hline & \multicolumn{1}{c|}{ Calcio } & \multicolumn{1}{c|}{ Zinc } & \multicolumn{1}{c|}{ Hierro } & Retinol & Tiamina & Riboflavina & Niacina & Vit C \\
\cline { 2 - 10 } & \multicolumn{1}{c|}{$5 \mathrm{mg}$} & \multicolumn{1}{c|}{$\mathrm{mg}$} & \multicolumn{1}{c|}{$\mu \mathrm{g}$} & \multicolumn{1}{c|}{$\mathrm{mg}$} & $\mathrm{mg}$ & $\mathrm{mg}$ & \multicolumn{1}{c|}{$\mathrm{mg}$} \\
\hline Requerimiento & \multicolumn{1}{c|}{5,1} & 5,8 & 400 & 0,5 & 0,5 & 6 & 30 \\
\hline $\begin{array}{l}\text { Alimento } \\
\text { complementario }\end{array}$ & 309,1 & 2,11 & 8,2 & 353,8 & 0,4 & 0,7 & 8,3 & 56,6 \\
\hline Lactancia materna & 386,7 & 0,86 & 0,4 & 310,4 & 0,2 & 0,3 & 0,5 & 12,7 \\
\hline Total & 695,8 & 3 & 8,6 & 664,2 & 0,6 & 1 & 8,8 & 69,3 \\
\hline \% Adecuación & 139,2 & 72,7 & 148,3 & 166 & 117,7 & 195,4 & 146,9 & 230,9 \\
\hline
\end{tabular}

El consumo de $10 \%$ a $20 \%$ de proteínas de origen animal, además de proveer aminoácidos esenciales, incrementa la oferta y la biodisponibilidad de minerales esenciales en la dieta (Dewey y Brown, 2003).

En las Tablas 3, 4, y 5 se muestran los requerimientos y el consumo de micronutrientes de los niños de 6 a 8, 9 a 11 y 12 a 36 meses de edad, respectivamente. En dichas Tablas de observa que para niños de 6 a 8 meses de edad se cubre sólo el 51,3\% de hierro y el $85,4 \%$ de zinc; en niños de 9 a 11 meses de edad, se cubre el 75,6\% de hierro y el 71,9\% de zinc. Para niños de 12 a 36 meses se llega a cubrir el 148,3\% de hierro, pero solo el $72,7 \%$ de zinc. El calcio y los demás micronutrientes como retinol, tiamina, riboflavina, niacina y vitamina $\mathrm{C}$, están cubiertos por las dietas en los tres grupos de edad estudiados.

El zinc en la dieta de los niños juega un papel muy importante en la prevención de la morbilidad y mortalidad causadas por las enfermedades infecciosas, pero también puede estar interfiriendo en el área de la visión debido a que el ion libre activa la enzima alcohol deshidrogenasa que cataliza la conversión de retinol a retinal. En el caso del hierro, los niños de seis a ocho meses de edad tienen dificultad para cubrir los requerimientos de este mineral a través de la alimentación complementaria.

El consumo de carnes como fuente de proteína es muy escaso en la dieta familiar de la población de Sarhua y precisamente el contenido y biodisponibilidad de zinc y hierro es alto en alimentos de origen animal, especialmente en la carne, vísceras y la yema de huevo. El consumo de vitamina $A$ excede al requerimiento en $22,5 \%$ para niños de 6 a 8 meses; 45,1\% para niños de 9 a 11 meses y $66 \%$ para niños de 12 a 36 meses. Al ser una vitamina liposoluble, su absorción está íntimamente relacionada con la presencia de lípidos; los ésteres de retinol disueltos en la grasa dietaria se dispersan en el intestino con la ayuda de las sales biliares (duodeno y yeyuno), por lo que cuando 
la dieta presenta un déficit en el consumo de lípidos, se afecta la biodisponibilidad de la vitamina A, pudiendo presentarse deficiencia de retinol sérico a pesar de haber suficiente consumo.

\section{Conclusiones}

La prevalencia de malnutrición crónica en los niños evaluados de 6 a 36 meses de edad del distrito de Sarhua fue de $55,8 \%$, de los cuales $34,9 \%$ presentaron malnutrición crónica moderada y 20,9 \% malnutrición crónica severa. No hubo malnutrición aguda, pero sí hubo tendencia a sobrepeso de 16,27 \%. Se observó baja adecuación de ingesta de micronutrientes tales como hierro y zinc. La prevalencia de anemia fue de $34,87 \%$ con una asociación significativa entre la edad y la gravedad de anemia, siendo mayor en el grupo de niños de 6 a 12 meses. El consumo de alimentos de origen animal (carne, pescado, huevo) fue muy escaso por lo cual se presenta una deficiencia de proteínas de alta calidad y de micronutrientes aunque el consumo de vitamina A excede a los requerimientos. Todos los niños evaluados recibieron lactancia materna exclusiva y luego continuada con alimentación complementaria, pero con deficiencia de alimentos de origen animal.

\section{Literatura citada}

Brown, J. y Pollit, E. 1996. Malnutrition, poverty and intellectual development. Scientific American, 274, 38-43. Centro Nacional de Alimentación y Nutrición [CENAN]. 2007. Tabla Peruana de Composición de Alimentos 2007. Microsoft Excel. 1 disco compacto, 8mm. Ministerio de Salud.

Dewey, KG.: Cohen, RJ.; Rivera, LL. y Brown, KH. 1998. Effects of age of introduction of complementary foods on iron status of breast-fed infants in Honduras. American Journal of Clinical Nutrition, 67, 878-874.

Dewey, KG. y Brown, KH. 2003. Update on Technical Issues Concerning Complementary Feeding of Young Children in Developing Countries and Implications for Intervention Programs. Food and Nutrition Bulletin, 24: 5-28.

Encuesta Demográfica y de Salud Familiar [ENDESINEI]. 2010.. ENDES, 2010. Ayacucho, Perú.

Organización de las Naciones Unidas para la Agricultura y la Alimentación [FAO]. 2004. Requerimiento de vitaminas y Minerales en Nutrición Humana, Informe de Consulta Mixta FAO/OMS de Expertos. Roma: FAO.

Instituto Nacional De Estadística E Informática (INEI). (2005). X Censo Nacional de Población y $V$ de vivienda. Ayacucho, pp. $35-40$.

Instituto Nacional de Salud [INS]. 2003. Encuesta nacional de consumo de alimentos en mujeres en edad fértil y niños menores de 12 a 35 meses. Informe Final. Lima.

Jason O'Neale, R. 2004. Lo Esencial en Metabolismo y Nutrición. (2da. edición). Madrid, España.

Leiva, B.; Inzunza, N.; Perez, H.; Castro, V.; Jansana, J. y Toro, T. 1991. Algunas consideraciones sobre el impacto de la desnutrición en el desarrollo cerebral, inteligencia y rendimiento escolar. Arch Latinoam Nutr. 51(1), 64-71.

Organización Mundial de la Salud [OMS]. 1995. El estado físico: uso e interpretación de la antropometría. Serie de Informes Técnicos, $N^{0}$ 854. Ginebra.

Presidencia del Consejo de Ministros (PCM). Secretaría Técnica de la Comisión Interministerial de Asuntos Sociales (ST-CIAS) Perú. (2004). Estrategia Nacional de Seguridad Alimentaria 2004-2015. Recuperado de http:// goo.gl/kk865r.

Pollit, E. 1997. Deficiencias de Hierro y Deficiencias Educacionales. Revisiones de Nutrición. 54: 133-140.

PRISMA. 1996. Medidas Caseras: Tabla de Medidas Caseras para la Programación y Evaluación de Regímenes Alimentarios. Lima: PRISMA.

World Health Organization [WHO]. 2001. Iron deficiency anemia. Assessment, prevention and control. A guide for program managers. Washington D.C.: WHO.

World Health Organization [WHO]. 2005 Anthro (Program of Computer). Anthropometric Calculator. Beta Version (Feb. 17 $7^{\text {th }} 2006$ ). 